\title{
ESTRATEGIAS PARA FOMENTAR UN ADECUADO COMPROMISO DEL FARMACÉUTICO CON RESPECTO A LA PUBLICIDAD DE MEDICAMENTOS DE VENTA LIBRE.
}

\section{STRATEGIES TO FOSTER AN ADEQUATE COMPROMISE OF THE PHARMACIST WITH RESPECT TO OVER THE COUNTER MEDICINES' ADVERTISEMENT.}

Pedro Domingo Armando ${ }^{1,4}$, María Isabel Tenllado², Patricia Martínez ${ }^{2}$, Sonia Andrea Naeko Uema ${ }^{3}$.

\author{
1 Doctor en Farmacia. Departamento de Farmacología. Facultad de Ciencias Químicas, Universidad Nacional de Córdoba. \\ 2 Farmacéutica. Colegio de Farmacéuticos de la Provincia de Córdoba. \\ 3 Doctora en Ciencias de la Salud. Departamento de Ciencias Farmacéuticas. Facultad de Ciencias Químicas, Universidad Nacional de Córdoba. \\ 4 Email de contacto: pdarmando@yahoo.es
}

\section{Conceptos clave}

Qué se sabe sobre el tema: la publicidad sobre medicamentos de venta libre (MVL) puede generar riesgos en la salud de la población si no es adecuadamente fiscalizada. Si bien se han realizado muchos estudios sobre el efecto de la publicidad de MVL en los consumidores, no existen demasiados trabajos con respecto a su impacto en las farmacias comunitarias.

Qué aporta este trabajo: la contribución de los farmacéuticos comunitarios permite disponer de estrategias profesionales individuales y colectivas para conseguir un empleo más racional de los MVL por parte de los pacientes.

\section{Resumen:}

Introducción: la publicidad ejerce una influencia muy importante sobre el público y, en el caso de los medicamentos de venta libre (MVL), puede generar riesgos en la salud de la población si no es adecuadamente fiscalizada. El objetivo de este trabajo es analizar y divulgar acciones estratégicas viables surgidas de la discusión en un foro de debate de un curso online, para fomentar el compromiso del farmacéutico ante la publicidad de MVL. Métodos: se realizó un análisis de contenido, agrupando por temas y categorías los aportes de los farmacéuticos comunitarios participantes en un foro de debate sobre publicidad de MVL. Como elemento disparador del debate, se analizaron 2 piezas publicitarias audiovisuales. Resultados: participaron 65 profesionales en 3 grupos de debate y se efectuaron 103 aportes. Dentro de las estrategias individuales se destacan la de garantizar la atención por un farmacéutico ante consultas sobre problemas de salud o sobre el uso de estos medicamentos. Otras estrategias incluyen denunciar ante la ANMAT las publicidades que no cumplan con las normativas vigentes, solicitar mejor fiscalización y asumir una postura crítica. Dentro de las estrategias colectivas se destacan: incluir a farmacéuticos en la comisión evaluadora de publicidad de la ANMAT e impulsar normativas para revalorizar el rol del farmacéutico y la farmacia comunitaria frente a los trastornos menores. Conclusión principal: la contribución de los farmacéuticos comunitarios permite disponer de un conjunto de estrategias profesionales individuales y colectivas para promover un uso más racional de los MVL por parte de los pacientes y la comunidad.

Palabras clave: medicamentos sin prescripción; publicidad directa al consumidor; política de salud; servicios comunitarios de farmacia.

\section{Abstract:}

The advertisement has a great influence on the public and, in over the counter medicines (OTC), it can generate health risks on the population if it is misregulated. The aim of this work is to analyze and disclose strategic viable actions emerged from a forum debate during an online course for fostering the pharmacist's compromise related to OTC advertisement. Methods: A content analysis was carried on, grouping by themes and categories the opinions of community pharmacists participating in a forum debate about OTC advertisement. Two audiovisual advertisement pieces were analyzed as triggers of discussion. Results: Sixty-five professionals participated in 3 groups of debate, and 103 opinions were collected. Among individual strategies, to guarantee the attention by a pharmacist was highlighted when health conditions or the use of medicines are consulted about. Other strategies include denouncing to the ANMAT the advertisements that do not comply with the norms, procuring a better regulation and assuming a critical posture. Within the collective strategies, including pharmacists in the evaluation committee of advertisement of ANMAT and furthering norms to reassess the role of the pharmacist and the community pharmacy in minor illnesses were stressed. Conclusion: The contribution of the community pharmacists allows disposing a set of professional strategies, both individuals and collectives, to promote a rational use of OTC for the patients and the community.

Keywords: nonprescription drugs; direct-to-consumer advertising; health policy; community pharmacy services. 


\section{Introducción}

Los medicamentos se diferencian, a partir de los riesgos que conlleva su uso, en medicamentos de venta bajo receta y medicamentos de venta libre $(\mathrm{MVL})^{1}$. En Argentina, la ley Nacional de Medicamentos № 16.463 y su Decreto Reglamentario 9763/1964 establecen que le corresponde la condición de venta libre a aquellos medicamentos destinados a aliviar dolencias que no exigen en la práctica una intervención médica y que, además, su uso en la forma, condiciones y dosis previstas, no entrañan por su amplio margen de seguridad, peligros para el usuario ${ }^{2,3}$. La Administración Nacional de Medicamentos, Alimentos y Tecnología Médica (ANMAT) es quien tiene a su cargo el registro de los MVL comercializados. Además, controla, fiscaliza y aprueba la venta de estos fármacos ${ }^{4}$.

La publicidad ejerce una influencia muy importante sobre el público y, en el caso de los medicamentos de venta libre (MVL), puede generar riesgos en la salud de la población si no es adecuadamente fiscalizada $^{5,6}$. La Organización Mundial de la Salud (OMS) establece que los anuncios dirigidos al público deben contribuir a que la población pueda tomar decisiones racionales sobre la utilización de los $\mathrm{MVL}^{7-9}$.

La ANMAT creó en 1994 una Comisión Evaluadora de Publicidad y Propaganda, que se encarga de analizar los anuncios de productos de uso medicinal, odontológico y cosmético, así como de los suplementos dietarios y tecnología médica. Los criterios para aprobar o rechazar las pautas presentadas por las empresas se basan en las normas vigentes en la materia. La Ley № 16.463 ya establece la prohibición de inducir a la automedicación en los anuncios de los MVL y esta norma fue complementada con otras más específicas, como la Resolución 20/2005 Ministerio de Salud de la Nación, la Disposición 4980/2005 de la ANMAT y posteriores ${ }^{10-12}$.

No obstante todas estas normativas, la realidad demuestra que el uso los medios masivos ha generado un exceso en la utilización de los productos farmacéuticos, favoreciendo la denominada "cultura de la píldora" ${ }^{13}$. Estudios efectuados en Argentina con avisos publicitarios emitidos por canales de televisión abierta y en horario central, ya han alertado sobre la gravedad del problema ${ }^{14,15}$. En este contexto, algunos autores coinciden en la necesidad de establecer estrategias para minimizar y contrarrestar estas situaciones que podrían tener efectos negativos para la salud por el uso inadecuado de los $\mathrm{MVL}^{16-18}$.

Si bien se han realizado muchos estudios sobre el efecto de la publicidad en los consumidores, no existen demasiados trabajos con respecto a su impacto en las farmacias comunitarias ${ }^{19}$. Algunos estudios han señalado que la publicidad de MVL atrae a los consumidores a adquirirlos sin importar el asesoramiento profesional, lo que compromete el papel del farmacéutico en la protección de los pacientes contra el uso inadecuado ${ }^{18}$. Esta publicidad puede promover también el autodiagnóstico y la automedicación; en consecuencia, es de interés público que las autoridades sanitarias apoyen y permitan a los farmacéuticos mantener su capacidad de proteger a la población frente al uso inadecuado de los MVL ${ }^{18}$.

Frente a la falta de estudios efectuados en el país para determinar la participación el profesional farmacéutico en esta problemática, se desarrolló el presente trabajo en el marco del curso on line "Gestión de Servicios Profesionales Farmacéuticos (SPF): indicación farmacéutica”, dictado por el Colegio de Farmacéuticos de Córdoba ${ }^{20}$. Este estudio tiene como objetivo analizar y divulgar acciones estratégicas viables surgidas de la discusión en un foro de debate de dicho curso, para fomentar el compromiso del farmacéutico ante la publicidad de MVL.

\section{Métodos}

Se realizó un análisis de contenido, agrupando por temas y categorías ${ }^{21-23}$, los aportes de los farmacéuticos comunitarios participantes en un foro de debate sobre publicidad de MVL, en el marco del curso on line "Gestión de Servicios Profesionales Farmacéuticos (SPF): indicación farmacéutica"20. Como elemento disparador del debate, se analizaron 2 piezas publicitarias audiovisuales ${ }^{24,25}$ que fueron seleccionadas específicamente por el equipo docente y en las cuales se observan incumplimientos de la normativa específica (Disp. ANMAT 4985/2005) ya descriptos por otros autores ${ }^{14}$.

Las consignas del foro de debate se centraron:

a) en el papel del profesional farmacéutico (tema 1) en la publicidad y en el cumplimiento de requisitos normativos (tema 2). 
b) en proponer estrategias (tema 3), tanto individuales como colectivas, para fomentar una adecuada intervención del farmacéutico en cuanto a la publicidad de los MVL.

La actividad tuvo una duración de 2 semanas (durante el mes de agosto de 2017) y, al finalizar, el docente responsable elaboró un resumen de los aportes. El texto elaborado fue enviado a través correo interno del curso a todos los participantes, con la intención de comunicar los resultados y permitir la incorporación de aportes desestimados o aclaraciones.

\section{Resultados}

Participaron 65 profesionales en 3 grupos de debate y se efectuaron 103 aportes.

Del análisis de contenido de las piezas publicitarias por temas, se destacan:

Tema 1: rol del profesional farmacéutico en la publicidad

- Figura del farmacéutico ausente o limitada a un "vendedor".

- Desprestigio del rol profesional.

Tema 2: cumplimiento de los requisitos normativos establecidos por la ANMAT

- No se atiende a la singularidad del paciente frente a un trastorno menor.

- Falta de información sobre el medicamento: posología, duración del tratamiento, efectos adversos, contraindicaciones, etc.

- Falta de recomendación de medidas no farmacológicas, ni promoción de hábitos saludables.

A continuación, se describen las estrategias individuales y colectivas, como tema 3 y subdivididos en categorías, señaladas por los farmacéuticos participantes para fomentar una adecuada intervención del farmacéutico en la publicidad de MVL. Las estrategias conjuntas incluyen ambos tipos de estrategias, ya que es posible realizarlas desde una única farmacia comunitaria, en conjunto con otras en una misma área geográfica 0 institucionalmente agrupadas. Las estrategias colectivas se relacionan básicamente a acciones a desarrollar desde los colegios y asociaciones profesionales o instituciones sanitarias.

Tema 3: estrategias profesionales frente a la publicidad de MVL

- Categoría estrategias individuales

- Garantizar la atención por un farmacéutico ante consultas sobre problemas de salud o uso de un MVL.

- Desarrollar el SPF de indicación farmacéutica, con registro y documentación.

- Evitar el uso de cartelería, exhibidores y chaquetillas con publicidad de MVL.

- No incentivar el uso indiscriminado de MVL. No emplearlos como vuelto por falta de cambio.

- Categoría estrategias conjuntas (individuales y colectivas)

- Denunciar publicidades que no cumplan con las normativas vigentes. Solicitar mejor fiscalización

y asumir una postura crítica. Unificar el formato y registrar denuncias y retroalimentaciones de ANMAT.

- Desarrollar campañas de concientización sobre el uso adecuado de MVL, con análisis crítico de la publicidad. Realizarlas en conjunto con otros colegios y asociaciones de profesionales sanitarios.

- Realizar talleres de educación sanitaria en la farmacia por categorías terapéuticas con pequeños grupos de pacientes. Organizar campañas y otras actividades educativas masivas desde los colegios profesionales, promoviendo la figura del farmacéutico como experto en medicamentos.

- Categoría estrategias colectivas

- Incluir a farmacéuticos en la Comisión Evaluadora de Publicidad de la ANMAT: representantes de colegios de farmacéuticos y universidades.

- Impulsar normativas desde los colegios profesionales: revalorizar el rol del farmacéutico y la farmacia frente a los trastornos menores. Evitar personajes mediáticos en las publicidades. 


\section{Discusión}

La publicidad de MVL basada en criterios de transparencia puede tener un papel beneficioso a nivel de la atención sanitaria y sobre la autonomía del paciente. Sin embargo, en virtud de su alcance muy poderoso y su intención de comercialización subyacente, puede llevar a los consumidores a adquirir medicamentos sin importar el asesoramiento profesional, lo que compromete el rol del farmacéutico para promover el uso racional de los medicamentos ${ }^{17,26}$. Todo ello desvirtúa la figura del farmacéutico o la limita a la de "simple vendedor", desprestigiando su rol profesional, tal como surge del análisis de las piezas publicitarias: en una mediante chistes entrega un medicamento y, en la otra, está ausente ${ }^{24,25}$. En ambos casos se induce al uso indiscriminado del medicamento, lo que conlleva a una automedicación irracional e inadecuada, en contraposición a las recomendaciones de los organismos internacionales ${ }^{7,14-16,27}$.

En otros estudios se ha reflejado la falta de poder de los farmacéuticos cuando se trata de pacientes expuestos a la publicidad, lo que no los hace susceptibles del consejo profesional. ${ }^{19}$ Los farmacéuticos comunitarios, como profesionales de la salud, son principalmente responsables del uso seguro y de calidad de los medicamentos, y tienen la responsabilidad ética de salvaguardar el bienestar de los consumidores ${ }^{17-19}$.

Del análisis de las piezas publicitarias se observó además que no se atiende a la singularidad del paciente frente a un trastorno menor y también la falta de información en las mismas sobre el medicamento (posología, duración del tratamiento, efectos adversos, contraindicaciones, etc). Tampoco se evidenciaron la recomendación de medidas no farmacológicas, ni la promoción de hábitos saludables ${ }^{8,17,18}$. Por ello se promueve como estrategia individual garantizar siempre la atención de un farmacéutico y el desarrollo del servicio profesional de indicación farmacéutica. Mediante esta prestación, el farmacéutico se responsabiliza de la elección de un MVL adecuado, con el objetivo de aliviar o resolver un problema de salud, si se trata de un trastorno menor, o la derivación del paciente al médico, cuando dicho problema necesite de su actuación ${ }^{20,28,29}$. Por ello, es de interés público que las autoridades apoyen y permitan a los farmacéuticos mantener su capacidad para proteger a la población contra el uso irracional de medicamentos ${ }^{7-19,26,27}$.

También se debe alentar a los farmacéuticos para que mejoren sus habilidades de comunicación y adquieran una comprensión sobre el equilibrio entre la autonomía del paciente y el juicio profesional en la práctica farmacéutica ${ }^{17-19,30}$. Esto se puede lograr mediante programas de capacitación personalizados diseñados por organismos profesionales o académicos ${ }^{17-19,26-30}$.

Como estrategias conjuntas, se señala la de conseguir una mejor fiscalización y asumir una postura crítica ante la publicidad, exigiendo a la ANMAT una vigilancia más estricta, tal como se planteó en otros estudios $^{14-15}$. Ya los trabajos realizados en la región de las Américas señalaban que hay escasa información sobre vigilancia y que no se contempla el papel de los consumidores ni de otras entidades en el monitoreo de las actividades promocionales ${ }^{14-15}$. Más concretamente, la Argentina mostró la tasa más baja de cumplimiento de las regulaciones, en particular con respecto a la mención de indicaciones no aprobadas $^{15}$. A causa de ello se confirma la función crucial que cumple la vigilancia activa de publicidad de MVL, así como también la necesidad de fortalecer las actividades de control y monitoreo ${ }^{14-16}$.

También como estrategias conjuntas se señalan el desarrollo de campañas de concientización sobre el uso adecuado de MVL, con análisis crítico de la publicidad en conjunto con otros profesionales sanitarios $^{8,15-17,19}$. Se resalta aquí el principio de cooperación de farmacéuticos y médicos para optimizar el buen uso de los medicamentos ${ }^{7,8}$. También se señalan como estrategias la de organizar campañas y otras actividades educativas masivas desde los colegios profesionales, para fomentar figura del farmacéutico como consultor en temas de farmacología y aproximar la farmacia comunitaria al sistema de salud en la práctica $^{7,29}$.

Con relación a estrategias colectivas se menciona la de incluir a farmacéuticos en la Comisión Evaluadora de Publicidad de la ANMAT, tanto representantes de colegios de farmacéuticos como de universidades. Este aspecto surge también de estudios anteriores, que resaltan la necesidad de fortalecer las actividades de control y monitoreo, lo que podría conseguirse por ejemplo mediante redes de apoyo de universidades, organizaciones no gubernamentales y otras entidades que trabajan en este campo ${ }^{14-19}$. 
Otra estrategia colectiva consiste en impulsar normativas desde los colegios profesionales que revaloricen el rol del farmacéutico y la farmacia comunitaria frente a los trastornos menores. Este aspecto también fue señalado en otras publicaciones que señalan que es de interés público que las autoridades apoyen y permitan a los farmacéuticos mantener su capacidad para proteger a la población contra el uso irracional de medicamentos ${ }^{19,27,29,30}$. Sobre este punto reviste particular interés la contra-publicidad de organismos gubernamentales y profesionales para minimizar la influencia de la publicidad como causante de un uso irracional de los medicamentos y la falta de poder del farmacéutico, promoviendo la búsqueda del consejo del farmacéutico comunitario ante la necesidad de un $\mathrm{MVL}^{15,19}$.

El análisis de la literatura permitió identificar otras estrategias que no fueron mencionadas por los farmacéuticos que participaron en el foro de discusión, tal como la necesidad de incluir en las regulaciones la rectificación pública cuando se comprueba que la información divulgada es engañosa o ambigua ${ }^{15,19}$. La contribución de los farmacéuticos participantes en el foro con sus aportes y opiniones permitió disponer de un documento de trabajo útil al momento de plantear estrategias.

Las estrategias individuales requieren de una actitud de compromiso profesional y de servicio a la comunidad por parte del farmacéutico. Las colectivas, además de surgir de la iniciativa personal, deben acompañarse con las instituciones o asociaciones profesionales y sanitarias para gestionar y coordinar las acciones pertinentes. Es necesario difundir las acciones estratégicas surgidas del foro de debate para lograr su concreción.

Limitaciones de responsabilidad: los autores declaran que los puntos de vista expresados en este manuscrito son de su responsabilidad y no de la institución en la que desempeñan sus actividades. No hubo financiación para el trabajo propiamente dicho.

Fuentes de apoyo: El Aula Virtual del Colegio de Farmacéuticos de Córdoba (https://colfacor.educativa.com/acceso.cgi?id_curso=) permitió desarrollar el curso y facilitó la discusión a través del foro de debate.

Agradecimiento: A todos los farmacéuticos comunitarios que participaron activamente en el foro de debate.

\section{Bibliografía}

1. Kregar G, Filinger E. ¿Qué Se Entiende Por Automedicación? Acta Farm Bonaerense. 2005;24(1):130-3.

2. Ley de Medicamentos, Ley Nacional 16.463, Poder Ejecutivo Nacional, República Argentina (Agosto 08, 1964).

3. Reglamentación de la Ley 16.463, Decreto № 9.763/1964 Poder Ejecutivo Nacional, República Argentina (Diciembre 07, 1964).

4. ANMAT. Publicidad: Requisitos básicos [Internet]. Buenos Aires: ANMAT, Ministerio de Salud de la Nación; c19962018 [acceso: 30/05/2018]. Disponible en: http://www.anmat.gov.ar/webanmat/publicidad/pautas_eticas.asp\#I_Medicamentos

5. ANMAT. Publicidad de Productos para la Salud [Internet]. Buenos Aires: ANMAT, Ministerio de Salud de la Nación; c1996-2018 [acceso: 30/05/2018]. Disponible en: http://www.anmat.gov.ar/comunicados/comunicado-publicidadconsumidores.pdf

6. ANMAT. Publicidad: Requisitos Éticos [Internet]. Buenos Aires: ANMAT, Ministerio de Salud de la Nación; c19962018 [acceso: 30/05/2018]. Disponible en: http://www.anmat.gov.ar/webanmat/publicidad/pautas_eticas.asp

7. Organización Mundial de la Salud. Criterios éticos de la OMS para la promoción de medicamentos (Resolución WHA 47.16).Ginebra: OMS; 1994.

8. Ramírez Puerta D, Larrubia Muñoz O, Escortell Mayor E, Martínez Martínez R La automedicación responsable, la publicidad farmacéutica y su marco en la Atención Primaria SEMERGEN. 2006;32(3):117-24.

9. ANMAT. Medicamentos de venta Libre: información que deben contener rótulos, prospectos y etiquetas [Internet]. Buenos Aires: ANMAT, Ministerio de Salud de la Nación; c1996-2018 [acceso: 30/05/2018]. Disponible en: http://www.anmat.gov.ar/comunicados/Prospectos-Nueva_Regulacion.pdf

10. Publicidad sobre productos de Venta Libre, Resolución 20/2005, Ministerio de Salud y Ambiente de la Nación, República Argentina (Enero 25, 2005).

11. Normas generales y específicas para publicidad de productos de Venta Libre, Disposición 4980/2005 Administración Nacional de Medicamentos, Alimentos y Tecnología Médica, Ministerio de Salud, República Argentina (Septiembre 09, 2005).

12. Comisión de Fiscalización y Control de Publicidad de productos de Venta Libre, Disposición 2335/2007 Administración Nacional de Medicamentos, Alimentos y Tecnología Médica, Ministerio de Salud, República Argentina (Mayo 02, 2007).

13. Barros JAC. Nuevas tendencias de la medicalización. Ciênc Saúde Coletiva. 2008;13 (Suppl): 579-58.

14. Aguzzi A, Virga C. Uso racional de medicamentos: La automedicación como consecuencia de la publicidad. Arch Venezolanos Farmacol Terap. 2009; 28(1):28-30.

15. Vacca $C$, Vargas $C$, Cañás $M$, Reveiz L. Publicidad y promoción de medicamentos: regulaciones y grado de acatamiento en cinco países de América Latina. Rev Panam Salud Pública. 2011;29(2):76-83. 
16. Pino D; Bedoya J, Correa M, Amariles $P$. Cumplimiento de la normatividad en la publicidad de medicamentos de venta libre en Colombia. Investigaciones Andina 2014;16(28):898-909.

17. Hassali MA, Al-Tamimi SK, Dawood OT. Consumer quality use of medicines: An important element in public health. Res Social Adm Pharm. 2017;13(1):261-265.

18. Chui MA, Stone JA, Martin BA, Croes KD, Thorpe JM. Safeguarding older adults from inappropriate over-thecounter medications: the role of community pharmacists. Gerontologist. 2014;54(6):989-1000.

19. Chaar B, Kwong K. Direct-to-consumer advertising: Australian pharmacists' experiences with non-prescription medicines. Int J Pharm Pract. 2010;18(1):43-50.

20. Colegio de Farmacéuticos de Córdoba. Curso on line Gestión de Servicios Profesionales Farmacéuticos (SPF): Parte 1: Indicación Farmacéutica [Internet]. Córdoba: Colegio de Farmacéuticos de Córdoba [acceso: 30/05/2018]. Disponible en: https://colfacor.educativa.com (acceso restringido).

21. Hancock B, Windridge K, Ockleford E. An Introduction to Qualitative Research. The NIHR RDS EM / YH, 2007.

22. Centers for Disease Control and Prevention. Analyzing Qualitative Data for Evaluation [Internet]. Evaluation Briefs 19. EEUU: CDC, Department of Health and Human Services; 2009 [acceso: 30/05/2018]. Disponible en: https://www.cdc.gov/healthyyouth/evaluation/pdf/brief19.pdf

23. Cavaco AM, Dias JP, Bates IP. Consumers' perceptions of community pharmacy in Portugal: a qualitative exploratory study. Pharm World Sci. 2005;27(1):54-60.

24. Sertal ${ }^{\circ}$ - Navidad. Video YouTube [Internet] [acceso: 23/11/2017]. Disponible en: https://www.youtube.com/watch?v=6SuHNPTaUel

25. Tafirol® - AdrianSuar "Chueco". Video YouTube [Internet] [acceso: 23/11/2017]. Disponible en: https://www.youtube.com/watch?v=hv4-xjs YtMQ

26. Organización Mundial de la Salud. Uso racional de los medicamentos: progresos realizados en la aplicación de la estrategia farmacéutica de la OMS. Reunión 118 del Consejo Ejecutivo, Informe de la Secretaría. Reporte No EB118/6; 2006.

27. Federación Internacional Farmacéutica, Organización Mundial de la Salud. Directrices Conjuntas FIP/OMS sobre Buenas Prácticas en Farmacia: Estándares para la calidad de los servicios farmacéuticos. Hyderabad: FIP/OMS; 2011.

28. Schimmelfing JT, Brookhart AL, Fountain KMB, Goode JKR. Pharmacist intervention in patient selection of nonprescription and self-care products. J Am Pharm Assoc 2017;57(1):86-89.

29. Organización Panamericana de la Salud, Organización Mundial de la Salud. Servicios farmacéuticos basados en la atención primaria de salud. SERIE La Renovación de la Atención Primaria de Salud en las Américas N 6. Washington DC: OPS; 2013.

30. van Eikenhorst $L$, Salema NE, Anderson C. A systematic review in select countries of the role of the pharmacist in consultations and sales of non-prescription medicines in community pharmacy. Res Social Adm Pharm. 2017;13(1):17-38. 